\title{
Men's perspectives of male hormonal contraception
}

\author{
Alison L. Lloyd ${ }^{1}$, Jackie Waterfield ${ }^{2} *$ \\ ${ }^{1}$ Centre for Clinical Practice, National Institute for Health and Care Excellence, Manchester, Lancashire, England \\ ${ }^{2}$ School of Health and Rehabilitation, Keele University, Staffordshire, England
}

Received: 14 June 2016

Accepted: 02 July 2016

\section{*Correspondence:}

Dr. Jackie Waterfield,

E-mail: j.waterfield@keele.ac.uk

Copyright: (C) the author(s), publisher and licensee Medip Academy. This is an open-access article distributed under the terms of the Creative Commons Attribution Non-Commercial License, which permits unrestricted non-commercial use, distribution, and reproduction in any medium, provided the original work is properly cited.

\section{ABSTRACT}

Background: In recent years there have been great developmental advances in male hormonal contraception (MHC). Despite this, research relating to men's perspectives of MHC is sparse and is usually based on questionnaires completed as part of clinical trials. This study explored men's perspectives of MHC, specifically how they were formed and what factors might be influencing them.

Methods: This qualitative study used semi-structured interviews with 10 heterosexual men aged between 18 and 44 within the UK. Using a philosophical standpoint of social constructionism, data were analysed employing a modified grounded theory method. Joint analysis and reflexivity were applied to reduce bias and ensure rigour in the analytical process.

Results: Four principal higher order themes emerged from the data: Sexual health and trust within a sexual relationship; Choice; Change; and Health. This paper presents the findings from the first two. Participants believed that MHC use would be affected by issues such as individual sexual relationships, sexual health, and trust. Issues relating to efficacy, contraceptive choice, age, knowledge and methods of administration were seen as core issues relating to the decision to take a MHC drug.

Conclusions: This study was successful in its aim, finding that overall MHC would be well received by men and that their perspectives were not that different from attitudes towards female hormone contraception. It also identified potential barriers based on the concerns that men have for themselves and for society were an MHC to become available.

Keywords: Male contraception, Men, Family planning, Perspectives, Attitudes

\section{INTRODUCTION}

Female hormonal contraception (FHC) and the subsequent social revolution first enabled the separation of sex and conception. ${ }^{1}$ However, options for men have not changed significantly in nearly a century, despite continued development of FHC.

In recent years, as a result of this contraceptive inequality ${ }^{2}$ there have been concerted efforts to develop male hormonal contraception (MHC). To date, clinical trials have identified 13 different methods that could potentially be used for this purpose. ${ }^{3}$ Of these, combined androgen and progestin hormones appear to be the most promising for general use. ${ }^{4}$

Despite public interest in this field, studies into the public's perspectives of MHC are sparse. Research has often only been undertaken through questionnaires completed alongside MHC clinical trials.

The social history and lifespan issues of FHC have some resonance with $\mathrm{MHC}$; for example, perceived existence of male inequities regarding their role in conception control. The social change caused by FHC, might be a predictor of similar changes for $\mathrm{t}$ MHCs with removal of 
this inequality, and may cause similar social change. Importantly, historical reflection on FHC cannot provide insights into the thoughts, feelings and background mind of the men affected by the change.

This study aimed to explore the perspectives men have of MHC, how these perspectives were formed, and what factors might be influencing them, in order to establish what issues may arise should an MHC become available.

\section{METHODS}

For this exploratory study a social constructionist approach was taken, allowing the researchers to examine men's views of the phenomenon of hormonal contraception through enquiry into their individual understandings and interpretations of the world., Understanding and perspectives of MHC are likely to be reflective of participants' existing knowledge and experiences, which in this case are unlikely to include direct contact with an MHC (as none are presently licensed for use).

Following ethical approval, participants were recruited from a population of male students and staff from a UK university through two different methods: direct recruitment by posters placed on noticeboards and emailing via the student and staff forum pages, and through snowballing via professional and personal contacts. To meet the aims of this study a sample of between 6-12 participants was considered. ${ }^{7}$ The final number was 11 (10 plus a pilot interview) and was based on achieving thematic saturation (see table 1). Inclusion/exclusion criteria were based on previous MHC clinical trials; transgender men were excluded. ${ }^{8}$ These criteria were chosen as the study required a sample to whom MHC would be directly rather than indirectly relevant.

After an information leaflet and consent form had been sent, mutually convenient semi-structured individual interviews were arranged, mostly conducted on the University campus, audio-recorded, and lasting no more than one hour.

An interview guide including topics such as relationships, heath and age was developed from relevant literature and from the researchers' expertise. Demographic data were collected at the beginning of the interview and at its conclusion the main discussion points were summarised as a form of respondent validation and to give the participant the opportunity to expand on areas discussed. ${ }^{9}$ Participants were given a numerical identifier to maintain anonymity. The lead researcher, AL, undertook all the interviews and transcription.

A pilot interview was carried out to assess and review feasibility, methods, timing and the interview guide, and to ensure that the data generated addressed the aims of the research.
Interviews and analysis were conducted in parallel over a 6-week period, allowing for emergent themes to be explored within subsequent interviews. Data analysis was performed using a modified grounded theory method ${ }^{10}$ through NVivo and any notes generated from the interviews were filed alongside the corresponding transcript.

Each transcript was read, coded and reviewed. This process involved going back to the original transcripts to ensure that the coding reflected the context from which it was drawn. This was repeated in the development of categories (a collection of codes that were similar in content), themes (a collection of categories that were similar and added to the overall concept) and higherorder themes (these were collections of themes sharing similar characteristics that captured the essence of participants' understanding and perspectives). The constant comparative method ${ }^{11}$ utilised throughout the analysis ensured rigour in the analysis and aided in a deeper understanding of concepts related to MHC being drawn from the data.

Additionally, reflexivity was key throughout this research and included reflexive accounts prior to commencing the study to avoid bias from any pre-conceived ideas, feelings or perspectives. ${ }^{12,13}$ Additionally both researchers reviewed transcripts and data analysis, thereby creating an audit trail. ${ }^{14}$.

\section{RESULTS}

While four higher-order themes emerged from the analysis - Sexual health and trust within a sexual relationship; Choice; Change; and Health - this paper presents the findings from the first two.

The participants' demographic table can be seen in Table 1.

\section{Higher order theme: Sexual health and trust within a sexual relationship}

This higher-order theme encompassed findings around sexual relationships, including the length of a relationship, sexual health, and sexual behaviour and how these factors might impact on the use of an MHC and trust.

\section{Sexual relationships}

While some participants exclusively used condoms within a relationship, others felt that MHC would appeal to those in a long-term relationship by both enhancing sexual spontaneity and preventing pregnancy, as illustrated by the following quotations:

"Maybe better using them in long term relationships as a preventative measure for pregnancy." (P9; 54-56) 
"I'd be more likely to take it when you've been with somebody 10, 15, 20 years and you know it's gonna, you know it's gonna last." (P9; 466-467). However, one participant suggested that in more casual encounters its appeal was as contraceptive safeguard against failure of barrier methods.

"Even when you're using condoms you know they break." (P10; 439-440)
For many participants, concerns about MHC's inability to protect against sexually transmitted infections (STIs) and the effect that this could have on STI transmission rates were an issue:

"Would this give rise to greater cases of sexually transmitted diseases, possibly because people would then rely on this just for the sake of pregnancy not thinking about the sexually transmitted diseases?" (P5; 434-438)

Table 1: Participant demographics.

\begin{tabular}{|c|c|c|c|c|}
\hline Participant & Age & Employment & Relationship status & Children \\
\hline 1 & 24 & Medical student & Single & 0 \\
\hline 2 & 34 & Senior administrator & Co-habiting & 1 \\
\hline 3 & 37 & Graphical designer & Married & 0 \\
\hline 4 & 36 & Teacher & Married & 2 \\
\hline 5 & 37 & Graphical designer & Married & 2 \\
\hline 6 & 24 & Medical doctor & In a relationship & 0 \\
\hline 7 & 35 & Senior carer & Co-habiting & 0 \\
\hline 8 & 30 & Trainee teacher & Co-habiting & 1 \\
\hline 9 & 34 & Carer & Married & 2 \\
\hline 10 & 25 & Programmer & Co-habiting & 0 \\
\hline
\end{tabular}

\section{Trust}

A key consideration for participants was trusting partners' effective use of any hormonal contraceptives.

"There's a trust issue with blokes to women, I think there have been situations certainly where blokes have been told that a female might be on contraception and isn't and I think that goes both ways." (P8; 311-314)

"Would women trust blokes? I mean you've heard they wouldn't, is it a trust issue? I think it can be overcome, yeah, because the only person you've got to trust is yourself to take that pill or to have that jab." (P8; 323326)

The lack of observable evidence of FHC was discussed, with one participant saying that FHC has an "element of mystery" (P4; 105) relating to its effective use. So MHC in combination with FHC would add a layer of "in-built security" (P8; 327); if either contraceptive fails or is taken ineffectually, the other would protect against conception.

Fidelity within the relationship, be it casual or long-term, was also a factor for participants when considering possible contraceptive behaviour. Participants felt that trust in a faithful partner was important in the use of MHC due to risk of STIs through infidelity:

"I think I would probably be more likely to take it in a relationship than one - night stands because you do use protection for one-night stands whereas you wouldn't when you're in a relationship.” (P6; 37-39)
"Well you've got to trust the person you're with 'cause if they're sleeping around a lot then you don't know that that tablet that you're taking is stopping you getting the diseases that the other person might bring." (P9; 456-458)

\section{Higher order theme: choice}

This higher order theme encompasses the various factors that would impact on men's choice to use a MHC, including attitude and lifestyle and the level of men's knowledge and understanding.

General factors that impact on choice to use the male hormonal contraceptive.

Participants generally received the idea of an MHC positively, feeling that it would be an acceptable alternative to current methods. However, they would want assurance of its efficacy; one participant suggested that he would want regular sperm count testing to achieve this, while others stated that they would be satisfied regarding efficacy if $\mathrm{MHC}$ were available through their doctor. Additionally, a range of MHC usage options, analogous to the FHC choices available to women, would be preferable as this would allow for adjustments on both side-effects and lifestyle.

"I don't think a one pill fits all, I think that it would be beneficial to have choice on the market." (P8; 269-271)

Convenience was another consideration. An MHC that was active for 3-6 months per dose would be appealing and some participants stated that they would prefer to 
avoid MHC injections -related partly to needle phobia and partly to a loss of control arising from the irreversibility of injected medications. Reversible methods (e.g. implant) or short acting methods (e.g. oral pill) would ameliorate long-term medication effects and an implant would have the additional advantage of convenience.

"Would it be convenient for me to take a pill every day? Yes I think it an option it's fine. Would it be an implant? Which one would be easier if I compared the pill to the implant? I think implant would be easier 'cause I don't have to worry about it." (P1; 409-411)

Participants were divided when considering whether younger individuals would choose to use MHC. Half of the participants felt that MHC would not be appealing, as it does not protect against STIs, yet its appeal could be an extra layer of protection against pregnancy.

“This isn't going to prevent any sexually transmitted diseases or anything like that so I think as a young chap who's out and about you know doing certain things it probably wouldn't appeal to them so much." (P2; $127-$ 130)

"Typical young free and single demographic who like to go out and have a bit of fun I think they'd be quite open to the idea in terms of giving them an extra layer of protection.” (P8; 186-188)

Interestingly, some participants also felt that an MHC would enable men to share the contraceptive pressure with women.

"Only women have responsibility, you know to take the pill or the coil or the implant or whatever, but I think probably what we're seeing is it's becoming more of an equitable relationship." (P4; 154-157)

\section{Social attitudes towards men and family planning}

Some participants identified that social expectations concerning contraception and family planning differed between men and women. One participant suggested that while women are expected to be responsible for contraception, they also have the power over decisions when pregnancy occurs. Yet, as participant 8 discussed, there was a perception that men should have financial responsibility despite having no control following conception.

"You have got is a culture in the UK where this hasn't been helped by things like the Child Support Agency where the bloke is always seen to have the easier ride, and you know if there is an unwanted pregnancy and that pregnancy is kept then it's the bloke who has to finance through the CSA. The male is often made to be the scapegoat." (P8; 333-338)

\section{Lifestyle factors that affect whether to take contraceptive}

All but one of the participants commented on cost in their decision to use an MHC, comparing the cost of $\mathrm{MHC}$ against existing methods (both male and female), frequency of purchase, as well as the overall impact on their financial situation. Equivalent cost to FHC would be important to avoid inequality and unfairness and some felt that the NHS should support the cost of MHC in line with female contraceptives.

For the majority of participants, convenience of MHC access and compatibility with their lifestyles were important, with an expectation that MHC would be available through established access points for FHC (such as pharmacies or family planning clinics). Six participants said that access to the drug would need to be controlled, either through prescription from a doctor or through a pharmacist.

"You'd probably want it from a doctor or a chemist or a sort of reputable source." (P4; 296-298)

"All the drug thing for me is just, it's to get rid of all the hassle. If there's hassle at the other end of the drug taking then it probably would put me off." (P3; 223-226)

Most of the participants felt they would have to consider the risks and benefits before choosing to use an MHC, and would be more receptive if there were reduced probability of severe side-effects, such as loss of longterm fertility.

This was balanced by participants suggesting they were likely to use a contraceptive for relatively short periods of time (1-10 years), though it was acknowledged that this would depend on their situation. Three participants felt that being responsible for contraception would assist in the prevention of unplanned pregnancies, thus giving men equal choice of when to start a family.

"It's a shared experience you can, you can just you can be part of that and not just give all the responsibility to a lady who is taking the pill every single day, whatever type of it, and then suffering through the side-effects. It's unfair I think. So if there's another way of making it kind of equal I think people will be a little bit happier." (P2, 172-175)

\section{Level of understanding of contraceptive methods}

All participants had experience of current methods of contraception. However, their level of knowledge about FHC methods varied; such prior knowledge seemed to be a major source of information when considering MHC. However, they did feel that before making a choice regarding $\mathrm{MHC}$ it would be important to have a good understanding of how it works and the possible sideeffects. 
"If you understand the reasons why you're taking the, the tablets or whatever, when you know that there's going to be this side-effect there's less of a shock to you." (P9; 111-113)

Participant 11 expressed concern about his lack of understanding of the male hormonal cycle, and how sperm is produced. For this reason he was concerned about the impact an MHC would have on his hormonal cycle.

"I don't think really think there's been the same level of research into what's actually going on in, like, men's cycle, like if there is even a cycle cause there's all this kind of people saying that men have their periods and stuff they have their hormonal moments and stuff like that but they don't really know what's that's caused by." (P11, 62-66).

\section{DISCUSSION}

This study has illuminated some of the perspectives and attitudes that men hold in respect of MHC. Reflecting findings by Eberhardt et al, participants in this study suggested that established relationships are most suited to MHC due to the lack of protection that it provides against STIs. ${ }^{15}$ The assumption that established relationships are less risky than casual encounters is interesting. In one sense this seems logical; fewer partners imply lower risk. However, there are two situations in which STIs may be transmitted between people within established relationships; partners may have undetected STIs that predate the relationship, or partners may have sex with others while maintaining their current relationship.

Most participants suggested that the risk posed by casual sex meant that they would always use condoms. However, there is little difference in terms of risk between casual and new relationships; unless STI screening has been conducted prior to sex without condoms within a new relationship, the risk may be similar to that of a casual sexual encounter. Flood's study suggests that condoms are commonly exchanged for FHCs early in relationships and this shift is described as demonstrating trust that a partner is faithful. ${ }^{16-18}$ The social convention of fidelity within established relationships and the sense of security that this brings could blind people to the risk of STIs from their partner. However, although it has been shown that the risks of STIs do not disappear in established relationships, it still seems reasonable to suggest that the risk is reduced when compared to sexual activity outside such relationships.

While the fact that condoms protect against both pregnancy and STIs was reported as the main advantage of the method, participants also discussed the possibility that MHC would result in an increase of STI transmission rates. They suggested this could be due to a greater concern about pregnancy than about STI transmission. Flood's participants also felt they were more likely to be involved in a conception than in obtaining an STI. ${ }^{16}$ This finding is interesting as it contradicts the participants' self-assessment of their risk calculation. Many of the participants in our study stated that they would always use condoms when having casual sex. However, the prevailing sense both in this and in Flood's study is that 'other people' are unlikely to take such care. ${ }^{16}$ On this basis it is difficult to know which would be the actual behaviour of the participants; and as a consequence participants' predictions of increased STI transmission may be based on their possible previous sexual behaviour. An alternative explanation for this discrepancy in belief is that, since the introduction of the Sexual Health Strategy, sexual health has become a prominent issue socially, within the NHS and the media. $^{23}$ Perhaps this increased exposure to STI information has resulted in the participants' perception that even though they themselves are not unsafe, others are irresponsible.

Sharing contraceptive responsibility within established relationships was discussed, with most participants feeling that the introduction of an MHC would increase equality within a relationship, offering an opportunity for men to share or fully assume the burden of contraceptive responsibility and side effects. This finding is consistent with previous studies, and perhaps the reason participants feel an MHC would increase equality is because there is presently no analogous alternative to the FHC. ${ }^{15,19-22}$ It is arguable that condoms already provide this opportunity to share responsibility, as agreement has to be reached between both individuals to use them, yet condoms are widely reported as an undesirable method due to perceived inconvenience and loss of sexual sensation. ${ }^{16}$ However, with current FHC methods (as would be the case with MHC), the decision to use it is often a personal choice and independent of consideration of others.

A significant additional significant finding was that participants suggested that MHC usage preferences may change dependent on age, with older men being considered less likely to use MHC. Their reasoning was that older men tend to have older partners who may have gone through the menopause. This is not necessarily the case, and thus some older men may continue to want to use an MHC. However, it is possible that these men may opt for vasectomy instead, especially if they have fathered as many children as they want to. The advantage of MHC for some men is its reversibility, which perhaps for the reasons above older men would not desire.

\section{Trust}

Trust was raised by a number of participants, with comments being focussed on two elements: trust within the relationship and trust in the efficacy of the MHC. Trust within the relationship related to men's concerns regarding the risk of unplanned pregnancies, specifically the trust that they have to put in a woman to manage contraception. This issue was raised by one participant 
reporting friends who were misled by women who said they were taking a contraceptive; however this situation is not limited to casual sexual encounters.

This mistrust is not specific to men; it appears to be a common basis of women's opposition to MHCs. ${ }^{19,24,25}$ As noted earlier, Flood found that condoms are exchanged for FHCs early in a relationship in those cases where men trust both their partner and the contraceptive method. ${ }^{16}$ However, the literature suggests that this would not be the case for MHCs, as women are reportedly less likely to trust men to be responsible for contraception. ${ }^{15,19,22}$ Some of the participants within this study said that they used condoms as their only form of contraception within their relationship. This suggests that their partners do trust them with this responsibility. Glasier et al shared this view; they showed that only $2 \%$ of their large, crosscultural female sample $(n=1894)$ would not trust their partner to take an $\mathrm{MHC} .^{26}$ In circumstances where trust was not certain, the administration route may provide sufficient evidence to satisfy a partner. Drugs given as either an injection or an implant would be self-evidential, allaying partners concerns regarding effective adherence to the medication.

The issue of mistrust of both the efficacy of MHC and men's management of contraception seems to be present equally in men and women. A major factor in this may be the novelty of this method. Some participants suggested that an MHC would replace FHCs in their relationships. Others suggested that they would prefer to take it alongside their partner's FHC. Meriggiola found that only $38 \%$ of their male sample would assume full contraceptive responsibility. ${ }^{20}$ One participant in our study felt that the addition of an MHC would increase control over paternity. He felt that the addition of an MHC would remove the need for trust, as each individual could be responsible for their own contraception, reflecting walker's findings. ${ }^{19}$ The potential for independent complementary contraceptive methods could cause an equalisation in trust, possibly affecting the social relations surrounding family.

Some findings related to an overall inclination to minimise personal adaptation expressed as a desire for convenience; that, ideally wants $\mathrm{MHC}$ to fit their lifestyle. This desire may have been the basis of participants' expectations of accessing MHC via the existing routes for FHC. This seemed to relate to the flexibility that the range of these routes provided. Additionally, convenience remained an issue within preferences regarding the route of administration; this and control over the duration of effect of MHC (in case of undesirable effects) were important features of any such route. It was felt that implants and oral pills would be desirable to manage undesirable effects, but 3-6 monthly injections would not. Importantly, while other research has identified that preferences exist, it has not hitherto explored the reasons for these preferences.
Our findings on cost suggested that barriers to acceptance may arise if MHC were to be priced higher than FHC (which is presently a prescription exempt from charge in the UK). However, it must be noted that equality of cost would impose a financial burden on the NHS, and would also imply an equal priority on MHC and FHC in terms of social attitudes and public policy. This is particularly interesting, as participants stated that MHC would not bring with it a need for social change, though this view seemed to be related more to moral or social issues such as those that FHC faced at its introduction - than to changes at policy level.

In summary, the principal findings from this study were that men would use an MHC, and that they want it to be similar to existing FHCs. Nonetheless, participants raised a number of issues that may present barriers to its introduction and uptake if not addressed, and these have been discussed in detail. Though not expressed directly by participants, it appears that the introduction of an MHC has the potential to have a dramatic social impact. FHC is currently exempt from prescription charge in the UK, and the expectation would be for MHC to also be exempt. Therefore companies, government departments and NHS policy makers will need to consider carefully the price of any resultant drug. Potentially, any man could use an MHC and policy makers may therefore need to consider which male groups are targeted to ensure efficiency of expenditure within the NHS.

\section{CONCLUSION}

This study has provided an insight into men's perspectives of MHC. It has identified some of the practical desires that men hold regarding MHCs as well as some of the concerns that they would have for themselves and for society were they to become available. This research has also raised some interesting questions relating to male identity in regard to $\mathrm{MHC}$ and social implications of its release. Further research could usefully explore these issues further.

\section{ACKNOWLEDGEMENTS}

We would like to thank all the individuals who took part in this research. We would also like to thank Julius Sim and Vivien Niblett for who proof read and edited this paper before publication

Funding: No funding sources Conflict of interest: None declared

Ethical approval: School of Health and Rehabilitation, Student Project Ethics Committee, Keele University, approval received 22nd May 2013

\section{REFERENCES}

1. Benagiano G, Bastianelli C, Farris M. Contraception: A social revolution. European Journal of 
Contraception and Reproductive Health Care. 2007;12(1):3-12.

2. Nieschlag E. Clinical trials in male hormonal contraception. Contraception. 2010;82:457-70.

3. Mathew V, Bentwal B. Male contraception. Indian Journal of Endocrinology and Metabolism. 2012;16(6):910-7.

4. Anderson RA, Baird DT. Male contraception. Endocrine Reviews. 2002;23:735-62.

5. Welford C, Murphy K, Casey D. Demystifying nursing research terminology: Part 1. Nurse Researcher. 2011;18(4):38-43.

6. Mason J. Qualitative researching. $2^{\text {nd }}$ ed. London: SAGE Publications; 2005.

7. Guest G, Bunce A, Johnson L. How many interviews are enough? An experiment with data saturation and variability. Field Methods. 2006;18(1):59-82.

8. Mommers E, Kersemaekers WM, Elliesen J, Kepers M, Apter D, Behre et al. Male hormonal contraception: a double blind, placebo controlled study. Journal of Clinical Endocrinology and Metabolism. 2008;93(7):2572-80.

9. Sim J, Wright C. Research in healthcare: concepts designs and methods. Stanley Thornes, Cheltenham. 2000 .

10. Strauss AL, Corbin J. Basics of qualitative research: Techniques and procedures for developing grounded theory. $3^{\text {rd }}$ ed. London. Sage Publications; 2008.

11. Glaser BG. The constant comparative method of qualitative analysis. Social Problems. 1965;12(4):436-45.

12. Dey I. Qualitative data analysis: A user friendly guide for social scientists. London: Routledge. 1993.

13. Angen MJ. Evaluating interpretive inquiry: reviewing the validity debate and opening the dialogue. Qualitative Health Research. 2000;10:37395 .

14. Johnson R, Waterfield J. Making words count: the value of qualitative research. Physiotherapy research International. 2004;9:121-31.

15. Eberhardt J, van Werschet A, Meikle N. Attitudes towards the male contraceptive pill in men and women in casual and stable sexual relationships. Journal of Family Planning and Reproductive Healthcare. 2009;35(3):161-5.

16. Flood M. Lust, trust and latex: why young heterosexual men do not use condoms. Culture, Health and Sexuality. 2003;5(4):353-69.
17. Corbett M, Dickson-Gómez J, Hilario H, Weeks M. R. A little thing called love: condom use in high-risk primary heterosexual relationships. Perspectives on Sexual and Reproductive Health. 2009;41(4):218-24.

18. Prata N, Vahidnia F, Fraser A. Gender and relationship differences in condom use among 15-24year olds in Angola. International Family Planning Perspectives. 2005;31(4):192-9.

19. Walker S. Attitudes to a male contraceptive pill in a group of contraceptive users in the UK. Journal of Men's Health. 2011;8(4):267-73.

20. Meriggiola MC, Cerpolini S, Bremner WJ, Mbizvo MT, Vogelsong KM, Martorana G, et al. Acceptability of an injectable male contraceptive regimen of norethisterone enanthate and testosterone undecanoate for men. Human Reproduction. 2006;21(8):2033-40.

21. Martin CW, Anderson RA, Cheng L, Ho PC, van der Spuy Z, Smith, et al. Potential impact of hormonal male contraception: cross-cultural implications for development of novel preparations. Human Reproduction. 2000;15(3):637-45.

22. Ringheim K. Evidence for the acceptability of an injectable hormonal method for men. Family Planning Perspectives. 1995;27(3):123-8.

23. Department of Health. The National Strategy for Sexual Health and HIV. London. Department of Health, HMSO.2001.

24. Campo-Engelstein L. Raging hormones, domestic incompetence, and contraceptive indifference: narratives contributing to the perception that women do not trust men to use contraception. Culture, Health and Sexuality. 2013;15(3):283-95.

25. Darroch JE. Forum: The pill and men's involvement in contraception. Family Planning Perspectives. 2000;32(2): 1 .

26. Glasier AF, Anakwe R, Everington D, Martin CW, va der Spuy Z, Cheng L, et al. Would women trust their partners to use the male pill? Human Reproduction. 2000;14:646-9.

Cite this article as: Lloyd AL, Waterfield J. Men's perspectives of male hormonal contraception. Int $\mathbf{J}$ Reprod Contracept Obstet Gynecol 2016;5:2546-52. 